\title{
Arquitectura de integración para el desarrollo de un sistema de geo-recomendación para establecer puntos de venta
}

Edith Verdejo Palacios, Giner Alor Hernández, Cuauhtémoc Sánchez Ramírez, Susana Itzel Pérez Rodríguez, José Luis Sánchez Cervantes, Lisbeth Rodríguez Mazahua

Instituto Tecnológico de Orizaba, División de Estudios de Posgrado e Investigación, Orizaba, México

everdejo@acm.org, \{galor, csanchez, lrodriguez \}@itorizaba.edu.mx, jsanchezc@ito-depi.edu.mx,

\begin{abstract}
Resumen. Los sistemas de geo-recomendación presentan la capacidad de realizar recomendaciones de lugares a partir de los intereses de los usuarios. Esta característica es útil en la competencia comercial ya que permite analizar mejor el estudio de localizaciones del mercado. Actualmente se considera que un factor clave para obtener el éxito comercial es la ubicación de los negocios; de manera que a medida que sean más cercanas con respecto a la localización de sus clientes, mayores serán los ingresos de las empresas. En este artículo se propone el diseño de una arquitectura de integración para el desarrollo de un sistema de georecomendación para el establecimiento de puntos de venta. La arquitectura se basa en un diseño de capas donde las funcionalidades de sus componentes e interrelaciones están distribuidas para un mejor mantenimiento y escalabilidad. Como prueba de contexto se presenta un caso de estudio que permite describir la arquitectura propuesta.
\end{abstract}

Palabras clave: Geolocalización, sistemas de información geográfica, sistemas de recomendación.

\section{Integrational Architecture for Developing a Geo-recommender System for Establishing Points of Sale}

\begin{abstract}
The geo-recommendation systems have the ability to carry out recommendations of places according to users interests. This feature is useful in commercial domains because it allows analyzing the study of potential markets locations. Nowadays, the business locations is considered a main factor to achieve the business success; so the profits can be increased if the business is more closer with respect to the location of its customers. This paper proposes the design of an integration architecture for developing a geo-recommender system to locate points of sale. The architecture is based on a layered design where the functionality of its components and relationships are distributed for better
\end{abstract}


Edith Verdejo Palacios, Giner Alor Hernández, Cuauhtémoc Sánchez Ramírez, et al.

maintenance and scalability. In order to validate our proposal, we present a case study describing the proposed architecture.

Keywords: Geolocation, geographic information system, recommender systems.

\section{Introducción}

Los sistemas de geo-recomendación son un nuevo punto de vista de los mecanismos de recomendación ya que son capaces de ofrecer recomendaciones tomando en cuenta las ubicaciones geográficas del usuario y de los lugares [1]. La principal atracción que ofrecen los sistemas de geo-recomendación reside en combinar sistemas de recomendación con información geográfica, donde el ámbito principal es el de actividades de ocio [1, 2]. Recientemente se identificó que las empresas deben ofrecer sus servicios a los clientes de manera rápida y oportuna debido a sus atareados estilos de vida; de manera que la cercanía de la ubicación de un negocio con respecto a la localización de sus clientes tiene un factor clave en el éxito de un negocio, ya que implica riesgos de imagen corporativa y financiera de la empresa [3]; y la distancia entre el domicilio del comprador y la ubicación del vendedor conlleva a un gasto adicional de transporte y tiempo. De tal forma que en los últimos años algunas empresas realizan estudios de mercado para evaluar a la competencia y su posicionamiento; intentando captar el negocio de sus contrarios investigando al consumidor y al entorno que le rodea, a través del diseño de mejores estrategias para captar un mayor número de clientes y solventar la preocupación del comportamiento de sus ingresos y egresos $[4,5]$. Por otro lado, el empleo de los Sistemas de Información Geográfica (SIG) a través de la construcción de modelos geográficos con integración del entorno socioeconómico ha conformado un nuevo punto de vista para el estudio del mercado, el cual no ha sido explotado lo suficiente por las empresas. Pero estos estudios presentan la gran limitante de no extraer el conocimiento de las necesidades de las empresas para que estas logren explotarlo las veces que sea necesario con el fin de obtener u ofrecer recomendaciones de acuerdo a su comportamiento empresarial. En cambio, la utilización de sistemas de recomendación permite ofrecer recomendaciones afines al comportamiento de los usuarios a través de técnicas que permiten analizar conductas.

La combinación de los sistemas de recomendación y los sistemas de información geográfica permitiría desarrollar nuevos sistemas de información que ofrezcan recomendaciones a partir de características económicas y demográficas; por lo que este artículo plantea el desarrollo de una arquitectura de sistema de geo-recomendación que sirva como base para hacerle frente al problema de selección óptima que enfrentan las empresas para la ubicación de sus instalaciones.

La estructura de este artículo es como sigue: la sección 2 presenta el estado del arte referente a sistemas de geo-recomendación y enfoques basados en dinámica de sistemas. La sección 3 presenta la arquitectura de integración propuesta. La sección 4 presenta un caso de estudio como prueba de concepto del sistema de georecomendación. Finalmente, se presentan las conclusiones de este artículo, así como también el trabajo a futuro. 
Arquitectura de integración para el desarrollo de un sistema de geo-recomendación ...

\section{Estado del arte}

A continuación se presenta la revisión del estado del arte sobre los trabajos relevantes que están relacionados directa o indirectamente con la selección de ubicación. Por lo que se decidió clasificar los artículos de acuerdo a los que utilizan mecanismos de recomendación y Sistemas de Información Geográfica (SIG).

\subsection{Sistemas de recomendación en diferentes dominios}

Colombo et al. [1] desarrollaron un sistema de recomendación móvil híbrido sensible al contexto, en donde se descartan las películas que no están siendo presentadas en las salas de cine y desechan los horarios de las películas a las que el usuario es probable que no asista basado en la distancia entre la posición origen y destino a partir de la identificación de las preferencias del usuario. Noguera et al. [2] discutieron que los cambios en el turismo electrónico requieren que los servicios proporcionen a los usuarios información relevante de acuerdo a sus contextos físicos actuales, teniendo en cuenta los gustos y preferencias. De tal forma que se propuso la implementación móvil sensible al contexto 3D de los restaurantes de la provincia de Jaén, España. Li et al. [6] propusieron un sistema de recomendación basado en los cupones de descuento con el fin de promover los productos pertenecientes a las plataformas en línea; a través de la construcción de árboles para categorizar los productos y un tratamiento de los datos para construir la red de usuarios y recoger datos de comportamiento. Batet et al. [7] desarrollaron un sistema de recomendación para dispositivos móviles basado en agentes; donde se ofrecen recomendaciones sobre actividades cercanas e interesantes para el usuario. Yu et al. [8] propusieron un sistema de inferencia basado en servicios de ubicación y conocimiento, el cual busca construir el conocimiento a través una aplicación móvil y mediante esta información el sistema puede ofrecer recomendaciones.

\subsection{Sistemas de información geográfica aplicados en estudios ambientales y urbanos}

Castro et al. [9] tomaron el modelo genérico de epidemiología para entender, modelar y analizar por medio de la dinámica de sistemas los factores críticos en la propagación de epidemias y la ejecución en un SIG con el propósito de visualizar e interpretar las fluctuaciones de sanos, infectados y recuperados. Corner et al. [10] plantearon un estudio para conocer los efectos de la deposición de los residuos de las granjas de peces. El cual utilizó una combinación de hojas de cálculo y un SIG por medio de un módulo de dispersión. Vairavamoorthy et al. [11] plantearon la falta de una herramienta capaz de predecir los riesgos por la intrusión de agua proveniente de alcantarillas, drenajes y zanjas a los sistemas de distribución de agua por medio del desarrollo un software predictor de riesgos asociados a los sistemas de distribución de agua fundamentados en SIG. Radiarta et al. [12] presentaron una evaluación multicriterio basada en un SIG que utiliza datos de detección satelital y datos de verificación de campo para identificar los sitios más adecuados para el desarrollo de la producción de vieira japonesa. Xu y Volker [13] formularon un estudio sobre las áreas residenciales en el desarrollo urbano, el cuál consistió en el análisis del SIG y de los 
sistemas dinámicos (modelo y visualización 3D) y la visualización espacial en 2D. Por otra parte, Suarez et al. [14] indicaron que la competencia y el desempeño de las franquicias son afectados en parte por los factores de selección en la ubicación y la calidad de las instalaciones. De manera que se emplearon modelos de localización competitiva y herramientas SIG. De manera similar, Roig et al. [15] desarrollaron una metodología para el proceso de selección de puntos de venta, en donde se utiliza SIG para visualizar los datos espaciales que influyen en la toma de decisiones y al proceso de jerarquía analítica (AHP), el cual consiste en definir un modelo a través de los criterios asociados a la localización y las alternativas de ubicación mediante un análisis en la geodemanda y geocompetencia. Finalmente, Casillas et al. [16] propusieron un estudio para conocer la intensidad y la hora en que ocurre la isla urbana de calor, a través de la interpolación de temperaturas generadas en un SIG.

La Tabla 1 presenta un análisis comparativo de la literatura, tomando en cuenta los SIG, Sistemas de Recomendación y Dinámica de Sistemas.

Tabla 1. Análisis comparativo de la literatura.

\begin{tabular}{|c|c|c|c|c|}
\hline NU=No Utilizado & H=Híbrido & prido & \multicolumn{2}{|c|}{$\mathrm{BC}=$ Basado en Conocimiento } \\
\hline Artículo & Objetivo & $\begin{array}{l}\text { Sistema de } \\
\text { recomenda } \\
\text { ción }\end{array}$ & SIG & $\begin{array}{l}\text { Dinámica } \\
\text { de } \\
\text { Sistemas }\end{array}$ \\
\hline $\begin{array}{l}\text { Castro et al. } \\
\text { [9] }\end{array}$ & $\begin{array}{l}\text { Plantear, desarrollar y simular un } \\
\text { modelo epidemiológico usando } \\
\text { SIG y dinámica de sistemas. }\end{array}$ & $\mathrm{NU}$ & ArcGIS & $\mathrm{NE}$ \\
\hline $\begin{array}{l}\text { Corner et al. } \\
{[10]}\end{array}$ & $\begin{array}{l}\text { Analizar, diseñar y construir el } \\
\text { módulo de dispersión de } \\
\text { deshechos marinos basado en } \\
\text { técnicas de SIG }\end{array}$ & NU & $\begin{array}{l}\text { TerrSet } \\
\text { (IDRISI) }\end{array}$ & NU \\
\hline $\begin{array}{l}\text { Vairavamoort } \\
\text { hy et al. [ } 11]\end{array}$ & $\begin{array}{l}\text { Desarrollar un software predictor } \\
\text { de riesgos asociados a los } \\
\text { sistemas de distribución de agua } \\
\text { fundamentados en SIG. }\end{array}$ & NU & ArcGIS & $\mathrm{NU}$ \\
\hline $\begin{array}{l}\text { Radiarta et al. } \\
\text { [12] }\end{array}$ & $\begin{array}{l}\text { Construir un modelo de } \\
\text { evaluación multicriterio basado } \\
\text { en técnicas SIG. }\end{array}$ & $\mathrm{NU}$ & ArcGIS & NU \\
\hline Yu et al. [8] & $\begin{array}{l}\text { Desarrollo de un sistema de } \\
\text { recomendación a partir de la } \\
\text { construcción de conocimiento } \\
\text { colectivo. }\end{array}$ & $\begin{array}{l}\mathrm{H} \\
\mathrm{NE}\end{array}$ & UN & NU \\
\hline $\begin{array}{l}\text { Xu y Volker } \\
{[13]}\end{array}$ & $\begin{array}{l}\text { Desarrollar un GISSD en 3D para } \\
\text { la evaluación de la sostenibilidad } \\
\text { del desarrollo urbano residencial. }\end{array}$ & $\mathrm{NU}$ & ArcGIS & Vensim \\
\hline $\begin{array}{l}\text { Suarez et al. } \\
\text { [14] }\end{array}$ & $\begin{array}{l}\text { Diseñar y construir modelos y } \\
\text { herramientas de localización } \\
\text { óptima para franquicias } \\
\text { comerciales. }\end{array}$ & NU & ArcGIS & NU \\
\hline
\end{tabular}


Arquitectura de integración para el desarrollo de un sistema de geo-recomendación ...

\begin{tabular}{|c|c|c|c|c|}
\hline NU=No Utilizado & $\begin{array}{l}\mathrm{NE}=\text { No } \\
\text { Especificado }\end{array}$ & \multicolumn{3}{|c|}{$\mathrm{BC}=$ Basado en Conocimiento } \\
\hline Artículo & Objetivo & $\begin{array}{l}\text { Sistema de } \\
\text { recomenda } \\
\text { ción }\end{array}$ & SIG & $\begin{array}{l}\text { Dinámica } \\
\text { de } \\
\text { Sistemas }\end{array}$ \\
\hline Batet et al. [7] & $\begin{array}{l}\text { Desarrollo un sistema de } \\
\text { recomendación de películas } \\
\text { híbrido para dispositivos móviles } \\
\text { basado en agentes. }\end{array}$ & $\begin{array}{l}\mathrm{H} \\
\mathrm{NE}\end{array}$ & NU & NU \\
\hline $\begin{array}{l}\text { Noguera et al. } \\
{[2]}\end{array}$ & $\begin{array}{l}\text { Desarrollar e integrar un motor de } \\
\text { recomendación móvil híbrido } \\
\text { sensible a la ubicación con una } \\
\text { arquitectura 3D-SIG. }\end{array}$ & $\begin{array}{l}\mathrm{H} \\
\mathrm{NE}\end{array}$ & $\mathrm{NE}$ & NU \\
\hline Roig et al.[15] & $\begin{array}{l}\text { Desarrollar un método de } \\
\text { selección de puntos de ventas } \\
\text { fundamentado en SIG y el } \\
\text { proceso de jerarquía analítica. }\end{array}$ & NU & ArcGIS & NU \\
\hline $\begin{array}{l}\text { Casillas et al } \\
{[16]}\end{array}$ & $\begin{array}{l}\text { Aplicar y validar la técnica de } \\
\text { modelado dinámico en la } \\
\text { estimación de intensidad y hora } \\
\text { en que ocurre la isla urbana de } \\
\text { calor. }\end{array}$ & NU & $\begin{array}{l}\text { TerrSet } \\
\text { (IDRISI) }\end{array}$ & Stella \\
\hline Li et al. [6] & $\begin{array}{l}\text { Desarrollar un mecanismo de } \\
\text { recomendación para compras } \\
\text { grupales con cupones de } \\
\text { descuentos a través de análisis de } \\
\text { preferencia y ubicación } \\
\text { geográfica. }\end{array}$ & $\begin{array}{l}\mathrm{H} \\
\mathrm{NE}\end{array}$ & NU & NU \\
\hline $\begin{array}{l}\text { Colombo et al } \\
{[1]}\end{array}$ & $\begin{array}{l}\text { Crear un sistema móvil de } \\
\text { recomendación de funciones de } \\
\text { películas; sensible a la ubicación, } \\
\text { el tiempo y a los espectadores. }\end{array}$ & $\begin{array}{l}\mathrm{BC} \\
\mathrm{NE}\end{array}$ & NU & NU \\
\hline
\end{tabular}

Con base al análisis realizado en la presente se observa que existe la necesidad de desarrollar un sistema que permita ofrecer recomendaciones de puntos de venta integrando sistemas de recomendación con sistemas de información geográfica.

\section{Arquitectura de integración}

La arquitectura de integración presenta un enfoque basado en capas. Este tipo de diseño permite escalabilidad y mantenimiento debido a que sus tareas y responsabilidades se encuentran distribuidas. La Figura 1 presenta el esquema general de la arquitectura de integración propuesta. Cada capa tiene una función que se explica a continuación:

Capa de Presentación: La capa de presentación se encarga de actuar como medio de comunicación entre los resultados obtenidos por las demás capas para los usuarios. 


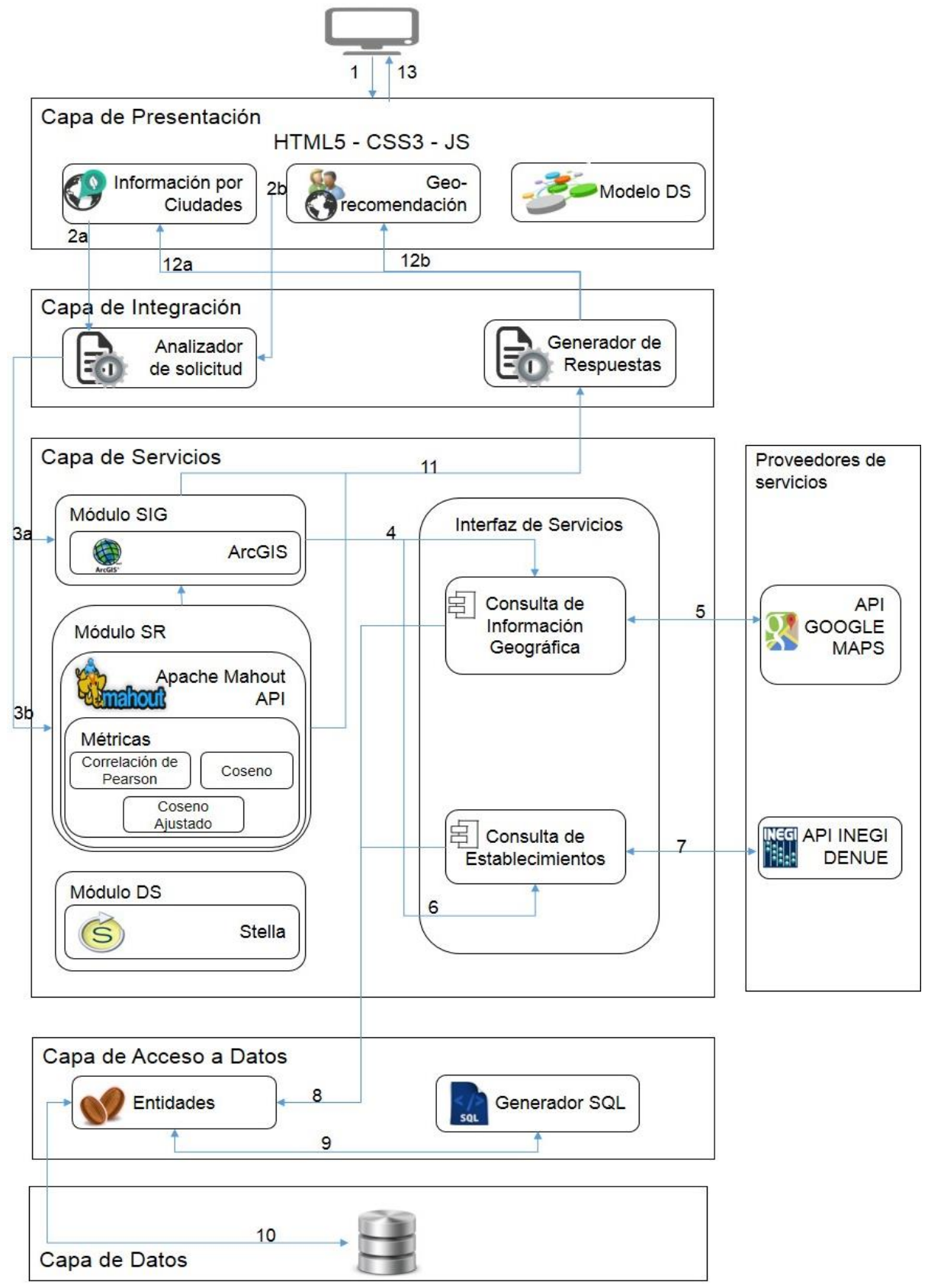

Fig. 1. Arquitectura del sistema de geo-recomendación.

Dentro de ella el usuario puede conocer la información de la ciudad, enviar la dirección del predio en forma de latitud y longitud para establecer un punto de venta; 
además de mostrar la recomendación final en formato de mapa y ver el modelo de dinámica de sistemas. Actúa como la interfaz, en donde el usuario podrá enviar la dirección del predio y recibir la recomendación en formato de mapa.

Capa de Integración: Esta capa permite re direccionar las solicitudes a los servicios que fueron solicitados en la capa de presentación. Así como la construcción de las respuestas.

Capa de Servicios: En esta capa se encuentra gran parte de operaciones con las cuales funciona el sistema. En ella se hallan los módulos de SIG, de recomendación, de dinámica de sistemas e interfaz de servicios. Es importante mencionar que esta capa realiza el trabajo de ofrecer la recomendación así como también permite tener la información necesaria de las restricciones relacionadas con los puntos de venta.

Proveedores de Servicios: Dentro de esta capa se encuentran las entidades que presentan los servicios de identificación de establecimientos y Geolocalización proporcionados por las APIs INEGI DENUE y Google Maps. El API INEGI DENUE permite consultar datos de identificación, ubicación y actividad económica a nivel nacional, por entidad federativa y municipio [17]. Google Maps es una API que ofrece un servicio Web de aplicaciones de mapas que pertenece a Alphabet Inc. Google Maps permite obtener la ubicación geográfica a partir de la devolución de un radio preciso de localización [18].

Capa de Acceso de Datos: La capa de acceso de datos se encarga de buscar y guardar la información en la Base de Datos que le solicita la capa de servicios. Es posible la encapsulación de tareas a través de las distintas entidades y la ejecución de las operaciones de inserción, eliminación, consulta y actualización por medio del generador de instrucciones basadas en SQL.

Capa de Datos: Esta capa almacena información acerca de las poblaciones, así como sus asentamientos y establecimientos (cines, escuelas, orfanatos, asilos, hospitales, templos, guarderías, mercados, auditorios, estadios y teatros)

En esta arquitectura, cada módulo tiene una función bien definida la cual se describe a continuación:

Módulo SIG: Este módulo es responsable de construir el modelo geográfico con base en las características geográficas obtenidas a partir de la consulta de las características geográficas de la zona y de establecimientos que son solicitadas a la interfaz de servicios.

Módulo de Recomendación (RS): Es el responsable de ofrecer las sugerencias al correlacionar el perfil del usuario con respecto a otros perfiles. Así como también es responsable de evaluar que la ubicación de un punto de venta es la adecuada. Pide el histórico de las demás recomendaciones a la capa de datos y por medio de las métricas de correlación de Pearson, coseno y coseno ajustado es capaz de ofrecer la georecomendación.

Módulo de Dinámica de Sistemas (DS): Inicializa el modelo con los factores socioeconómicos para poder iniciar la simulación de posibles escenarios, generando información estadística.

Interfaz de servicios Web: Permite conectar con los servicios Web de geolocalización y de información de establecimientos; posibilita la creación de contenidos completos por medio de combinar datos provenientes de distintos servicios Web. 


\section{Caso de estudio: sistema de geo-recomendación}

Para la validación de la arquitectura se plantearon los siguientes argumentos:

a) Una empresa desea establecer puntos de ventas en la ciudad de Orizaba, Ver. para comercializar su producto.

b) Es necesario que cada punto de venta cumpla con la regulación vigente perteneciente al Reglamento para las acciones de construcción, instalación, conservación y operación de estaciones de servicio en gasolinera y carburación.

c) La empresa desea automatizar el proceso de búsqueda de establecimientos para la colocación de puntos de venta.

Siendo estipulado lo anterior; ¿Cómo podría la empresa satisfacer las condiciones anteriores para establecer su punto de venta?

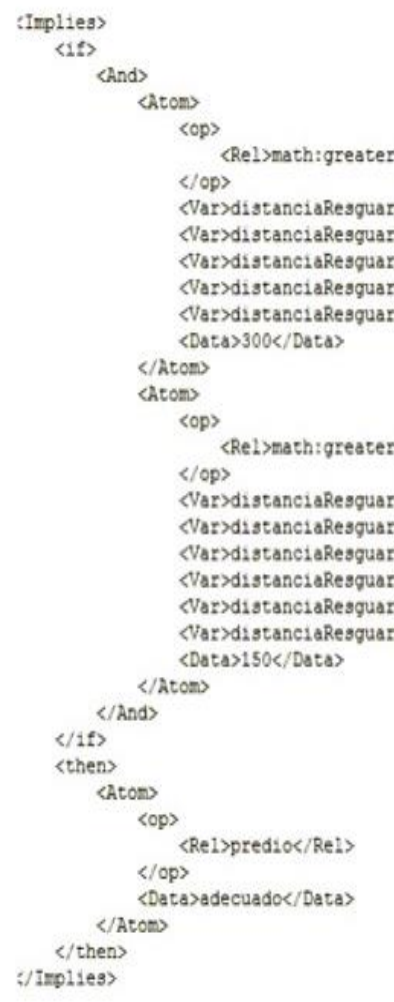

a)

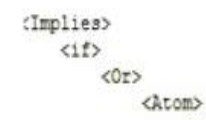

atoms

〈Op> Rel>math:LessThan</Re1>

〈/op>

〈Var>distanciaResquardoRadialHospital</Var> 〈ar>distanciaResguardoRadialisilo</Var> War>distanciaResquardoRadia10rfanato</Var> CVar>distanciaResquardoRadialGuarderia〈/Var〉 ¿War>distanciaRegquardoRadialEgcuela</Var) $\langle$ Data>300</Data>

$</$ Acoss

〈Atoms

$\langle 0 p\rangle$

$\langle/ o p\rangle$

War>digcaneiaResquardoRadiolMercado</Var) (Var>distanciaResguardoRadisiTemplo</Var) 〈Var>distanciaResguardoRadisLihuditoriok/Var〉 (War>distanciaResquardoRadiaiCine</Var > 〈War〉distanc1aResguardoRadiaiEgtadio</Var〉

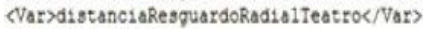
(Atoms 〈Data>150</Data> $\langle/ 0 r\rangle$ $\langle/ 19\rangle$ (thens $\langle$ Atom>

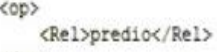
$\langle/ o p\rangle$ CDatasinadecuado</Datas </Atom mpliess

Fig. 2. a) Regla para predio adecuado, b) Regla para predio inadecuado.

Como primer paso, es necesario definir las reglas para la colocación de establecimientos las cuales se encuentran en el artículo 10 de la regulación 
anteriormente mencionada; la cual indica que el predio debe ubicarse a una distancia mínima de resguardo de 300 metros radiales de centros de concentración masiva, tales como escuelas, hospitales, orfanatos, guarderías, asilos; así como a 150 metros radiales de mercados, cines, teatros, estadios, auditorios y templos [19]. El principal motivo de contar con reglas es el hecho de tener especificaciones que ayuden a identificar áreas óptimas. Para la representación de las reglas fue necesario utilizar RuleML 1.0. RuleML es un lenguaje para reglas en formato XML que provee una manera de expresar las reglas de negocio [20]; a través de la técnica de expresión de reglas se presentan las condiciones y acciones (antecedentes y consecuentes) que derivan de ella.

La Figura 2 presenta las reglas para la identificación de predios adecuados e inadecuados. Estas restricciones de ubicación indican que las estaciones de servicio deben respetar cierta distancia con respecto a museos, escuelas, hospitales, orfanatos, guarderías, teatros, cines, auditorios y templos. Como segundo paso, por cada asentamiento se buscó su información geográfica (latitud y longitud) por medio de la conexión al API de Google Maps. Cabe mencionar que para obtener la información respecto a los establecimientos mencionados anteriormente fue necesario construir una llamada al servicio proporcionado por la API DENUE del INEGI y guardar la información de los establecimientos, así como también la construcción de un mecanismo para la actualización de la información.

Por el momento se cuenta con un prototipo de la aplicación Web basada en el Framework JSF con PrimeFaces. La Figura 3, muestra la visualización de la información obtenida a partir de las consultas a los servicios Web de las APIs previamente mencionadas.
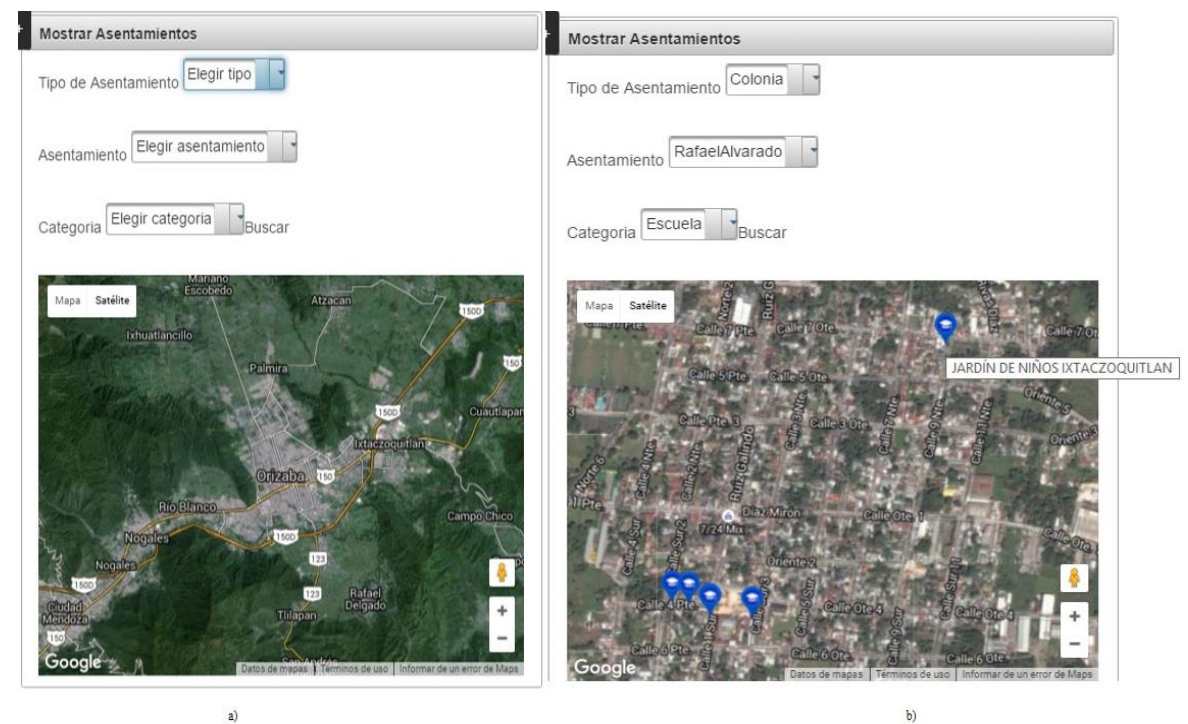

Fig. 3. Visualización de establecimientos.

Posteriormente, la Figura 4a muestra el formulario de búsqueda de puntos de venta, en él se introduce la dirección que se considera adecuada para establecer un punto de venta, en formato de longitud y latitud. En caso de no ser una ubicación 
adecuada de acuerdo con las restricciones del reglamento, especificará que la localización no es la adecuada. La Figura 4b, presenta los resultados de sugerencia de posibles puntos de venta, en caso de que la ubicación si cumpla con las restricciones del reglamento; mostrando en forma circular de color verde los sitios más óptimos, en naranja los sitios con una probabilidad media y en rojo los sitios menos adecuados.

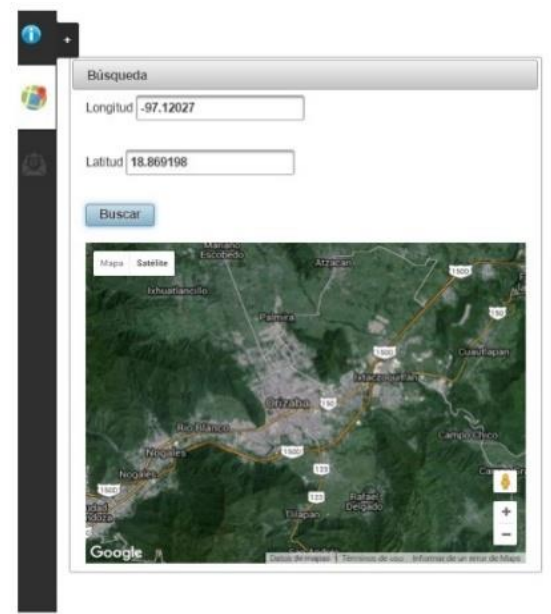

Fig. 4. a) Formulario de Búsqueda, b) Resultados.

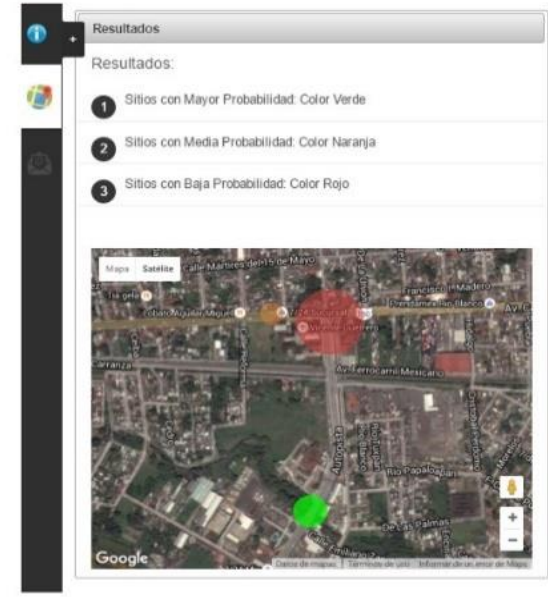

Finalmente, la Figura 5 presenta el detalle de la forma circular seleccionada, donde un porcentaje nos indica que tan recomendable es la zona para establecer puntos de venta.

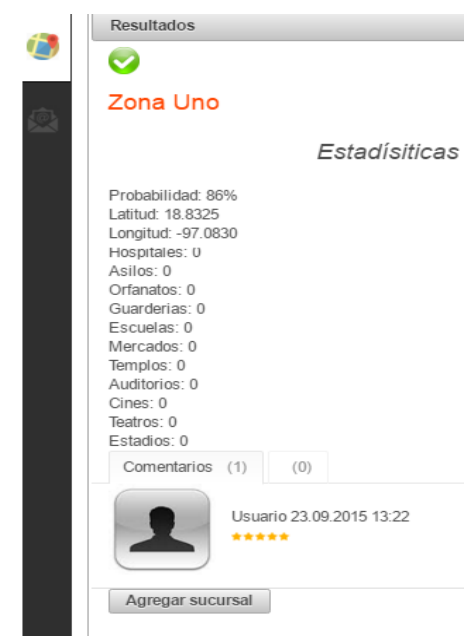

Fig. 5. Detalle de la zona.

Así como también la identificación de establecimientos cercanos (hospitales, asilos, guarderías, orfanatos, escuelas, mercados, templos, auditorios, cines, estadios y 
teatros). El prototipo permite visualizar el detalle de cada una de las tres zonas (Alta, Media y Baja) encontradas mostrando los porcentajes y la puntualización de las reglas. Para este ejemplo se muestra el detalle de una zona con mayor posibilidad de ser adecuada para la colocación de un punto de venta; así como también la especificación de cumplir con las reglas de distancia de resguardo al no encontrase con ningún establecimiento cercano.

En esta sección se aprecia un solo escenario, no obstante se estan probando otros escenarios como la búsqueda radial, búsqueda por calle y búsqueda por codigo postal. La búsqueda radial permite identificar a partir de un punto (longitud y latitud) y una distancia radial ubicaciones apropiadas para establecer un punto de venta; donde muchas veces las superficies de estas búsquedas implican más de un asentamiento derivando en evaluaciones de restricciones para cada uno de los puntos que integren dicha superficie y en algunas ocasiones encuentra más de un punto de venta optimo. La búsqueda por calle de un asentamiento posibilita hacer una evaluación de las restricciones a todos los puntos que integran el tamaño de una calle. Esta es una búsqueda muy similar al caso de estudio salvo que en esta el usuario selecciona el asentamiento y la calle para poder encontrar un punto de venta.Finalmente la búsqueda por código postal hace evaluaciones por todas las superficies de los asentamientos que pertenecen al código postal y al igual que la búsqueda radial en determinadas circustancias devuelve más de un punto de venta adecuado. De manera que en casos con más de un punto de venta optimo, la decisión depende del usuario.

\section{Conclusiones y trabajo futuro}

El uso independiente de modelos geográficos y dinámica de sistemas para el análisis del establecimiento de locales comerciales, son las tradicionales líneas de investigación que están sujetas a limitaciones; ya que las características socio-demográficas influyen en la estrategia de localización de las empresas. La integración de los sistemas de información geográfica y modelos dinámicos facilita en gran parte la creación de estrategias que ayudan en la toma de decisión de las empresas. Sin embargo, esos estudios no ofrecen las características necesarias para el futuro, por lo que la incorporación de los sistemas de recomendación, dinámica de sistemas y SIG permitirá ayudar a los comerciantes en la toma de decisiones con respecto a la ubicación, tener la seguridad de encontrar un mercado potencial que les ayude a mejorar sus ingresos. Además de que la propuesta de la arquitectura de integración plantea la fusión de tecnologías que aún no han sido publicados por otros estudios.

Como trabajo futuro se pretende completar el desarrollo de los módulos de dinámica de sistemas, de recomendación y SIG, del sistema de recomendación para la ubicación de establecimientos que permita identificar las áreas de la zona de localización óptima. Así como también incrementar el número de ciudades pertenecientes a la zona centro del Estado de Veracruz y el estudio en la regulación vigente para la inclusión de reglas.

Agradecimientos. Los autores agradecen el apoyado por el Consejo Nacional de Ciencia y Tecnología (CONACYT), Tecnológico Nacional de México (TecNM) y la Secretaria de Educación Pública (SEP) a través de PRODEP. Así como también al 
Instituto Nacional de Estadística e Informática (INEGI) por contar con APIs que permiten consultar la información del Territorio Mexicano.

\section{Referencias}

1. Colombo-Mendoza, L.O., Valencia-García, R., Rodríguez-Gonzélez, A., Alor-Hernández, G., Samper-Zapater, J.J.: RecomMetz: A context-aware knowledge-based mobile recommender system for movie showtimes. Expert Systems with Applications, Vol. 42, No. 3, pp. 1202-1222 (2015)

2. Noguera, J.M., Barranco, M.J., Segura, R.J., Martínez, L.: A mobile 3D-GIS hybrid recommender system for tourism. Information Sciences, Vol. 215, pp.37-52 (2012)

3. García-Palomares, J.C., Gutiérrez, J., Latorre, M.: Optimizing the location of stations in bikesharing programs: a GIS approach. Applied Geography, Vol. 35, No. 1, pp. 235-246 (2012)

4. Zolfani, S.H., Aghdaie, M.H., Derakhti, A., Zavadskas, E.K., Morshed Varzandeh, M.H.: Decision making on business issues with foresight perspective; an application of new hybrid MCDM model in shopping mall locating. Expert systems with applications, Vol. 40, No. 17, pp. 7111-712 (2013)

5. Piastria, F.: Avoiding cannibalisation and/or conipetitor reaction en planar single facility location. Journal of the operations research, Vol. 48, No. 2, pp. 148-157 (2005)

6. Li, Y.-M., Chou, Ch.-L., Lin, L.-F.: A social recommender mechanism for location-based group commerce. Information Sciences, Vol. 274, pp. 125-142 (2014)

7. Batet, M., Moreno, A., Sánchez, D., Isern, D., Valls, A.: Turist@: Agent-based personalised recommendation of tourist activities. Expert Systems with Applications, Vol. 39, No. 8, pp. 7319-7329 (2012)

8. Yu, Y-H. Kim, J-H., Shin, K., Jo, G.S.: Recommendation system using location-based ontology on wireless internet: An example of collective intelligence by using 'mashup'applications. Expert systems with applications, Vol. 36, No. 9, pp. 11675-11681 (2009)

9. Castro Castro, C.A., Londoño Ciro, L.A., Valdés Quintero, J.C.: Modelación y simulación computacional usando sistemas de información geográfica con dinámica de sistemas aplicados a fenómenos epidemiológicos. Revista facultad de ingenieria Universidad de Antioquia, Vol. 34, pp. 86-100 (2005)

10. Corner, R.A., Brooker, A.J., Telfer, T.C., Ross, L.G.: A fully integrated GIS-based model of particulate waste distribution from marine fish-cage sites. Aquaculture, Vol. 258, No. 1, pp. 299-311 (2006)

11. Vairavamoorthy, K., Yana, J., Galgalea, H.M., Gorantiwara, S.D.: IRA-WDS: A GIS-based risk analysis tool for water distribution systems. Environmental Modelling \& Software, Vol. 22, No. 7, pp. 951-965 (2007)

12. Radiarta, I.N., Saitoha, S-I., Miyazono, A.: GIS-based multi-criteria evaluation models for identifying suitable sites for Japanese scallop (Mizuhopecten yessoensis) aquaculture in Funka Bay, southwestern Hokkaido, Japan. Aquaculture, Vol. 284, No. 1, pp. 127-135 (2008)

13. Xu, Z., Coors, V.: Combining system dynamics model, GIS and 3D visualization in sustainability assessment of urban residential development. Building and Environment, Vol. 47, pp. 272-287 (2012)

14. Suárez-Vega, R., Santos-Peñate, D.R., Dorta-González, P.: Location models and GIS tools for retail site location. Applied Geography, Vol. 35, No.1, pp. 12-22 (2012) 
Arquitectura de integración para el desarrollo de un sistema de geo-recomendación ...

15. Roig-Tierno, N., Baviera-Puig, A., Buitrago-Vera, J., Mas-Verdu, F.: The retail site location decision process using GIS and the analytical hierarchy process. Applied Geography, Vol. 40, pp. 191-198 (2013)

16. Casillas-Higuera, Á., García-Cueto, R., Leyva-Camacho, O., Gonzalez-Navarro, F.F.: Detección de la Isla Urbana de Calor mediante Modelado Dinámico en Mexicali, BC, México. Información tecnológica, Vol. 25, No.1, pp. 139-150 (2014)

17. http://www.inegi.org.mx/desarrolladores/denue/apidenue.aspx

18. https://developers.google.com/maps/documentation/geolocation/intro

19. Boley, H., Paschke, A., Shafiq, O.: RuleML 1.0: the overarching specification of web rules. Lecture Notes in Computer Science, 6403(4), pp. 162-178 (2010)

20. H. Ayuntamiento de Córdoba, Ver. Reglamento Para Las Acciones De Construcción, Instalación, Conservación y Operación De Estaciones De Servicio En Gasolinera y Carburación para el Municipio de Córdoba, Ver, pp. 3-4 (2014) 